\title{
La educación y la cultura: una ruta hacia las dos caras del perfil docente
}

\author{
Education and culture: a route towards the two faces of the teaching profile \\ Educação e cultura: um caminho para as duas faces do perfil docente
}

\section{ARTÍCULO ORIGINAL}

\begin{tabular}{|c|c|c|c|c|}
\hline 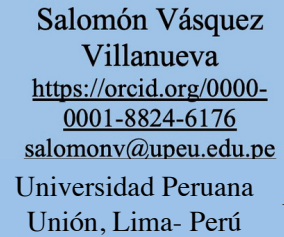 & $\begin{array}{l}\begin{array}{c}\text { Salomón Axel Vásquez } \\
\text { Campos }\end{array} \\
\text { https://orcid.org/0000-0001- } \\
\text { 9405-0794 } \\
\text { salomonvasquez@upeu.edu.pe } \\
\text { iversidad Peruana Unión, Lima- Perú }\end{array}$ & $\begin{array}{c}\text { Marina del Águila } \\
\text { Gonzales de del Castillo } \\
\text { https://orcid.org/0000-0003- } \\
\underline{2343-4533} \\
\text { marina010454@,gmail.com } \\
\text { Universidad Nacional de la } \\
\text { Amazonía Peruana, Iquitos- Perú }\end{array}$ & 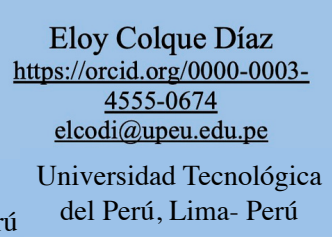 & $\begin{array}{c}\begin{array}{c}\text { Denix José Deza } \\
\text { Arrestegui }\end{array} \\
\text { https://orcid.org/0000-0002- } \\
\underline{8255-0481} \\
\text { dede@upeu.edu.pe } \\
\text { Universidad Peruana } \\
\text { Unión, Lima- Perú }\end{array}$ \\
\hline
\end{tabular}

Recibido 10 de Enero 2022 | Arbitrado y aceptado 17 de Junio 2022 | Publicado en 11 Octubre 2022

\section{RESUMEN}

El perfil del docente ha sido y siempre será una preocupación importante, para los beneficios de la educación y el proceso enseñanza-aprendizaje. En este sentido, el estudio tiene el objetivo de determinar las dimensiones del perfil educativo y cultural del docente. Para lograr este objetivo se ha revisado los estudios relacionados con este perfil, motivo de la investigación. Se concluye que el perfil educativo y cultural del docente tiene las dimensiones: educación holística, inclusiva, acreditada, gamificada, emotiva e investigativa, para la variable perfil educativo; para la variable perfil cultural, entre otras, visión de diversidad cultural, de difusión cultural, de identidad cultural, de cultura innovadora y cultura organizacional.

Palabras clave: perfil docente, educación, cultura.

\section{ABSTRACT}

The teacher's profile has been and will always be an important concern for the benefits of education and the teachinglearning process. In this sense, the study aims to determine the dimensions of the educational and cultural profile of the teacher. To achieve this objective, the studies related to this profile, the reason for the research, have been reviewed. It is concluded that the educational and cultural profile of the teacher has the dimensions: holistic, inclusive, accredited, gamified, emotional and investigative education, for the educational profile variable; for the variable cultural profile, among others, vision of cultural diversity, cultural diffusion, cultural identity, innovative culture and organizational culture.

Keywords: teacher profile, education, culture.

\section{RESUMO}

O perfil do professor foi e sempre será uma preocupação importante para os benefícios da formação e do processo ensino-aprendizagem. Nesse sentido, o estudo visa determinar as dimensões do perfil educacional e cultural do professor. Para atingir esse objetivo, foram revisados os estudos relacionados a esse perfil, motivo da pesquisa. Conclui-se que o perfil educacional e cultural do professor possui as dimensões: formação holística, inclusiva, credenciada, gamificada, emocional e investigativa, para a variável perfil educacional; pelo perfil cultural variável, entre outros, visão da diversidade cultural, difusão cultural, identidade cultural, cultura inovadora e cultura organizacional.

Palavras-chave: perfil do professor, educação, cultura. 
Salomón Vásquez, Salomón Axel Vásquez, Marina del Águila Gonzales, Eloy Colque, Denix José Deza Arrestegui

\section{Introducción}

Las inquietudes académicas, profesionales e investigativas, sobre el perfil del docente han sido, son y serán permanentes. El perfil del docente no se ha desligado del tiempo. Estas inquietudes se han constituido en una constante en las universidades, en el ámbito de las autoridades educativas, incluso, en los más altos niveles de los poderes del Estado. No es una temática de reflexión que pertenece a este tiempo, sino de todos, inclusive han quedado marcados los hitos en la historia de la educación nacional e internacional. Sin embargo, no se ha determinado con mucha precisión, exactitud, objetividad y convencionalidad los contenidos, las dimensiones del perfil docente, el cual por su propia naturaleza ya es complejo, arbitrario y controvertido.

El perfil docente, en su dimensión educativa, implica la práctica de una educación holística, en el sentido del desarrollo del hombre en todos los aspectos, cuya visión y perspectivas son exclusivas de los docentes, quienes participan y actúan sin discriminación, más bien con la mirada de inclusión, sin dejar al margen la acreditación educativa, de la cual no se puede prescindir por las exigencias y rigores académicos e institucionales, inmersos en las políticas educativas globales.

El docente desarrolla su intervención en ámbitos, donde se perciban la diversidad, la identidad, la difusión cultural. El docente si identidad carece de valor y expectativa; no sirve de ejemplo para los demás, particularmente para sus discípulos, porque el docente no solamente enseña, sino modela y modera los comportamientos; es paradigma para las generaciones. Respondiendo a estas inquietudes, se pretende determinar el perfil educativo y cultural del docente, en un contexto de mejorar la cosmovisión de los pueblos y la sociedad respecto de la presencia y el rol del docente.

2. El perfil educativo y cultural del docente

2.1 Perfil educativo del docente

\section{A) Reflexiones conceptuales}

Para Galvis, Fernández y Valdivieso (2006, citados por Galvis, 2007), el perfil docente es "el conjunto de competencias organizadas por unidades de competencias, requeridas para realizar una actividad profesional, de acuerdo con criterios valorativos y parámetros de calidad" (pp. 52 y 53).

Importa mucho la definición del perfil docente. Los logros institucionales dependen del buen desempeño de los docentes. La calidad de las instituciones no queda desligada de la actuación de los docentes. El perfil de los docentes debe quedar orientado hacia el fortalecimiento de las instituciones educativas. La gestión académico-administrativas 
define el perfil del docente. La educación es un instrumento, un factor muy importante, determinante, de influencia poderosa, de avance y progreso para las personas, la sociedad y las instituciones, comprometidas con el conocimiento, la cultura, los valores, el espíritu (Dimas-Rangel y Treviño-Sánchez, 2015).

Estos conceptos presentan una constante de términos: conocimientos, capacidades, competencias, habilidades, destrezas. Sobre la base de estos conceptos se construye el perfil académico y cultural del docente, a luz del estudio de Lozano-González (2017).

Una de las características del perfil docente es la autoestima: "protectora de la salud mental", muy importante para los protagonistas en el ámbito educativo, predice el éxito académico, evita el estrés. Los docentes deben trabajar el desarrollo de las competencias socioemocionales en los estudiantes (Jiménez-Hernández, Sancho-Requena y SánchezFuentes, 2019). Según Alsina, Batllori, Falgàs y Vidal (2019), la construcción del perfil doce tiene doce marcas. Las primeras cinco son: "experiencias previas; creencias sobre uno mismo; creencias sobre el funcionamiento de la clase; conocimientos disciplinares implícitos; y conocimientos didácticos implícitos"; las siete marcas restantes son: “interacción con el contexto I (el centro escolar); interacción con el contexto II (la universidad); interacción con uno mismo; interacción entre iguales; interacción con el experto; interacción con la teoría; y conocimiento profesional crítico".

B) Dimensiones del perfil educativo del docente

La educación holística

Hernández-Mosqueda, Tobón-Tobón y Vázquez-Antonio (2014), por su parte, abordan la docencia socioformativa, en el contexto de la construcción y consolidación de la sociedad del conocimiento, que busca, procesa, crea, adapta y aplica el conocimiento, con el propósito de encontrar y proponer la solución de los problemas locales, parciales, de acuerdo con una visión global, sin el aislamiento de las TICs. La socioformación se ha constituido en un enfoque con el fin de formar personas integrales, sin perder de vista lo ético, el trabajo colaborativo/equipo, el emprendimiento y la gestión del conocimiento, mediante la aplicación las competencias requeridas "para identificar, interpretar, argumentar y resolver los problemas de su contexto con una visión global y a través de proyectos interdisciplinarios" (Tobón, 2013a, 2013b, citado por Hernández-Mosqueda et al., 2014, p. 90).

En el ámbito educativo, queda matriculada la perspectiva holística; es decir, la participación docente, la intervención del mismo, el enfoque y la misión del docente 
tienen una naturaleza holística, aborda al ser humano en toda su plenitud, busca el desarrollo pleno, integral y holístico de la persona. En este sentido,

La esencia en la formación docente es el desarrollo de cualidades creativas, estratégicas, científicas, emocionales, estéticas y valores como la responsabilidad, el respeto, la gratitud, la justicia, la honradez, etc. La comprensión de la naturaleza de las relaciones humanas, resolución de conflictos y capacidad de diálogo, constituyen factor importante (Barrientos-Gutiérrez, 2016, p. 172).

La educación inclusiva

¿Qué significa la educación inclusiva? Arroyo-González (2013) sostiene que la educación intercultural se ha constituido en el camino que conduce hacia la inclusión educativa. Los escenarios donde actúan los docentes son espacios donde la educación intercultural y la inclusión educativa se encuentran, donde se dan la mano, cuyos actores protagonizan y escenifican sus acciones en pro de la renovación de las prácticas educativas y la organización escolar. Ambos conceptos ofrecen múltiples conexiones que guían la práctica educativa.

Por su parte, Florian (2014) expresa que al inicio la educación inclusiva se enfrentó a críticas diversas sobre los múltiples problemas conexos y sobre los grandes desafíos inmersos, en la educación especial, hoy llamada educación inclusiva, dejando en manos de los docentes y las autoridades educativas el reto de reimaginar su nuevo rol, la reestructuración de la calidad de la educación para todos, las capacidades de los aprendizajes, los derechos iguales para todos, la búsqueda de la homogeneidad en medio de la heterogeneidad, mediante el establecimiento y la práctica de una pedagogía inclusiva.

La educación inclusiva qué revela, qué dice del docente. La educación inclusiva tiene relación con la realidad educativa crítica, con las necesidades educativas especiales ante la falta de especialistas, responde a un enfoque curricular compensatorio, deficitario. Significa construir nuevas cosmovisiones, cambios educativos, transformaciones de mentalidades sociales, para atender y satisfacer necesidades de la sociedad en fragmentos menores. Semánticamente, la educación inclusiva ha sustituido a la educación especial, cuyas características son: holística, abierta, compleja, en una perspectiva de concepción y prácticas educativas, sistema escolar moderno, cambio y transformación de medios y materiales de aprendizajes (Leiva-Olivencia \& Gómez-Gerdel, 2015).

Se busca, fomenta y difunde una educación que decline, evite y margine el racismo discriminatorio. Debe trabajar agendas, programas y políticas educativas, dejando al 
margen el racismo, presente en los espacios sociales, políticos, educativos, culturales; inclusive en las aulas y los recintos escolares. La educación restablece, vindica a los hombres y las mujeres, cuya procedencia se ubica en las sociedades aborígenes de Latinoamérica (Velasco-Cruz, 2016).

Se requieren buenas prácticas en educación inclusiva, dejando al margen las experiencias excluyentes, también las divergentes y contradictorias, propiciando el desarrollo de los modelos nacionales e internacionales. Se busca los consensos en las propuestas, los trabajos, las investigaciones, los objetivos, los valores, los modelos, la equidad, la calidad, las claves de la educación inclusiva, las circunstancias sociales, de género, origen étnico, antecedentes familiares. Es una respuesta de calidad a la diversidad étnica, cultural, educativa, social, lingüística, con enfoques equitativos, compensatorios, asistenciales, focalizados hacia la globalidad (Muntaner-Guasp, Rosselló-Ramón y De La IglesiaMayol, 2016). Durante los dos últimos años, las modalidades de educación: a distancia y virtual, en este tiempo, se han constituido en una estrategia para la educación inclusiva en América Latina; el concepto de educación inclusiva ha sido modificado (Maturana y Mahecha, 2020). Esta concepción implica la recuperación de los derechos educativos de los hombres, de las mujeres, de los niños, de las niñas, quienes se muestran con discapacidad, con culturas diferentes, desplazados y marginados, otorgándoles una "admisión parcial o completa en los diferentes niveles del sistema educativo" (Clavijo y Bautista, 2020).

La educación acreditada

La evaluación y la acreditación, si son consistentes y a largo plazo, conducen a las universidades hacia los estándares de la excelencia académica; son necesarias e imprescindibles para generar el fortalecimiento y el reconocimiento de los niveles de calidad, en todas sus dimensiones. La acreditación constituye en un medio muy importante para el reconocimiento de la educación superior; permite validar los procesos y sus calidades; se ha formalizado en el camino, en la vía para que el Estado reconozca si la calidad de las universidades es buena (Acosta y Acosta, 2016).

Las instituciones educativas, las autoridades y los docentes se movilizan dentro y fuera de los escenarios educativos en busca de la acreditación educativa, cuya tendencia es universal, porque se busca la calidad educativa y reconocida por las instituciones educativas y políticas nacionales e internacionales, garantizando de esta manera su permanencia, vigencia y reconocimiento. Se necesita garantizar los procesos educativos de calidad, ante los diversos organismos externos nacionales e internacionales. Implica 
renovar los procesos curriculares, las prácticas, los aprendizajes, las evaluaciones, particularmente en las instituciones de educación superior (Martínez-Iñiguez, Tobón y Romero-Sandoval, 2017).

Todas las instituciones se mueven en búsqueda de la acreditación educativa, porque se precisa que la acreditación educativa genera impacto positivo y significativo sobre la mejora de los programas que ofrece la institución, la universidad. La evaluación y la acreditación constituyen la acción importante de las instituciones que buscan mantenerse en el espacio y el tiempo. La acreditación es un proceso de evaluación, que implica el reconocimiento público, también se ha constituido en una estrategia de panificación, permite la gestión de la calidad, el logro de la exceptivas; significa la validación de los reconocimientos; la evaluación y la acreditación forman variables transversales (Márquez y Zeballos-Pinto, 2017).

La acreditación jamás aparece al margen de la evaluación. Tienen una mutua dependencia. Las dos coexisten. Ambas requieren de jueces externos, a más de los internos. Son herramientas eficaces, revelan la mejora de los sistemas educativos. Se busca la acreditación de los programas educativos. La calidad de los programas depende de la evaluación y la acreditación otorgada por organismos externos a las instituciones educativas (Jiménez, 2019).

La gamificación educativa

Es denominada también gamificación en educación. Es una estrategia didáctica, que permite la integración de los juegos y su dinámica en contextos no lúdicos, con el propósito de potenciar la motivación y los valores positivos de los juegos. Se propone la construcción de un currículo orientado hacia la gamificación de la educación, cuyos principios están generando muchos y significativos beneficios para los aprendizajes de los estudiantes. La gamificación contribuye hacia el logro del compromiso y permite alcanzar el denominado engagement. Permite el uso de videojuegos, mecánicas de juegos, cuyo objetivo es la motivación (Ortiz-Colón, Jordán y Agredal, 2018).

Corchuelo-Rodriguez (2018) aborda la gamificación con el sentido de una estrategia docente que busca la motivación de los estudiantes y la dinámica del desarrollo de los contenidos, en el espacio específico denominado aula. Es considerada una herramienta que transforma la educación, gracias a Pelling, quien en 2002 acuñó el término por primera vez, en el sentido de adaptar el juego en el espacio de la educación, sin dejar al margen el uso de las TICs. Esta estrategia o herramienta sirve para realizar la motivación, el mejoramiento de las dinámicas grupales, la atención, la crítica reflexiva y, sobre todo, 
el aprendizaje significativo. Para Werbach y Hunter (2012, citados por CorchueloRodriguez, 2018), la gamificación exitosa presenta 6 elementos:

1) Definir con claridad los objetivos educativos que se quieren conseguir en el aula, de esta manera, se diseñan con coherencia y eficacia las estrategias de gamificación. 2) Delimitar los comportamientos que queremos potenciar en los estudiantes como conocimientos, actitudes, habilidades, entre otros. 3) Establecer quienes son los jugadores, identificar rasgos y características para diseñar actividades pertinentes a sus intereses reales. 4) Establecer los ciclos de las actividades, definiendo el sistema de gamificación (mecánicas de juego, orden de los eventos, interacción, entre otros). 5) Diversión (es la base de todo juego), se deben describir los eventos de este tipo que se incluyen en la estrategia. 6) Recursos, incluye las herramientas que se van a usar para el desarrollo de la estrategia (medición, seguimiento, indicadores, etc.) (p. 30).

La gamificación y el aprendizaje afectivo, en entornos virtuales, buscan el establecimiento de un ambiente amigable, cuya respuesta corresponde a los usuarios y sus respectivas necesidades emotivas, dejando al margen los "escenarios fríos y rígidos", características propias y exclusivas de estos entornos. Tienen el propósito de despertar la incitación y la motivación comprometidas con la construcción del conocimiento y el desarrollo de las competencias de los estudiantes (Melo-Solarte y Díaz, 2018).

Para Parra-González y Segura-Robles (2019), la gamificación no es sino una metodología emergente, la cual utiliza los juegos y las mecánicas del mismo, en contextos no lúdicos, con el propósito de cultivar la motivación de los estudiantes en escenarios educativos, inclusive en escenarios científicos. En este sentido, se ha constituido en una herramienta poderosa para la motivación de los estudiantes en el aula y agenciar el proceso enseñanzaaprendizaje mucho más eficiente y eficaz. En la parte de su concepción, aparecen ligados los conceptos: metodología, metodología emergente, herramienta, recurso emergente e innovador.

La educación emotiva

Para Bisquerra y Hernández (2017), el docente busca, estimula, fomenta y desarrolla el bienestar de los estudiantes; imparte la educación emocional; conoce y practica la psicología positiva; el docente inteligente construye aulas felices, actúa en medio de las mismas. El bienestar integral es la meta de la educación y de las políticas educativas. La educación emotiva implica y compromete la búsqueda del bienestar económico, material, social, tecnológico, profesional, psicológico, emocional, hedónico, eudemónico. Son 
necesarias y pertinentes la educación emotiva y la cultura del bienestar. La educación emocional construye el bienestar; implica la generación de una atención plena.

Melgar y De Los Reyes Leoz (2018) abordan las experiencias educativas emotivas y creativas. Significa educar y cultivar las emociones, la sensibilidad. La educación tiene este compromiso. Las vivencias emotivas inspiran, estimulan la comprensión, el disfrute, la voluntad de escuchar y hablar. Es necesaria y pertinente la interacción emocional, también la generación de emociones en contextos sociales, socioculturales, sentimentales. Las emociones vinculan a las personas con la familia, con las instituciones, con los objetos.

Los procesos de enseñanza y aprendizaje guardan relación directa con la inteligencia emocional y las experiencias emocionales o emotivas, sin dejar al margen la racionalidad. Es decir, las emociones se juntan con la racionalidad durante la educación de las personas. Se pretende la psicologización de la educación, en el sentido de entender que la Psicología positiva constituye una ciencia esencial muy importante para la educación, porque facilita el conocimiento y los aprendizajes, el bienestar subjetivo denominado emociones positivas (Prieto-Egido, 2018).

Para Moreto, González-Blasco y Piñero (2018), la educación afectiva se genera mediante el cultivo y la práctica de la empatía, las emociones y los recursos pedagógicos. La empatía facilita la construcción de las relaciones, es un factor fundamental, muy importante. En este sentido, se busca, se fomenta una educación de la afectividad, de las emociones y de los sentimientos, en el contexto y la dependencia de la empatía, que significa el aprecio de los sentimientos de los otros, los demás.

La educación investigativa

La universidad cumple tres misiones: "la enseñanza y formación superior; la investigación y generación de conocimiento; así como el servicio a la sociedad mediante el más amplio impacto cultural, social y económico de su actividad en la construcción de una sociedad mejor, más justa y democrática" (Díez-Gutiérrez, 2018). De acuerdo con Smith y Smith (2018), en las instituciones educativas falta la investigación en educación, con el propósito de generar los cambios sustantivos en la educación, haciéndola más sostenible, evidente y objetiva para todos. Para Corredor \& Saker (2018), el docente ha sido formado con la perspectiva de investigador, lo que significa conocer y dominar la aplicación de métodos de investigación científica. Es decir, las competencias científicas de los docentes comprometen la capacidad de vínculos con los métodos utilizados en la investigación científica, con las capacidades de análisis. El docente, con capacidades de 
investigación, no solamente ha sido formado con esta perspectiva, sino conoce y domina el universo de la investigación, además, lo más importante, trasmite los conocimientos y las prácticas investigativas a sus estudiantes, quienes se constituyen en discípulos en el campo de la investigación.

Para Montes y Mendoza (2018), la docencia y la investigación caminan juntas en el espacio y los ámbitos académicos, especialmente en la universidad, en el entendido de que ambas exigen una valoración económica. En este sentido, el Estado y las universidades quedan obligadas a establecer políticas relacionadas con la docencia y la investigación. Inclusive se encuentra una trilogía comprometida con la investigación: Estado, empresas y universidades. Para la investigación se requiere presupuesto, infraestructura, equipamiento, tecnología e investigadores expertos, cuyas influencias vienen de docentes formados en universidades instaladas en países desarrollados.

La docencia y la investigación son consolidadas en la universidad, generando la presencia de docentes-investigadores. La investigación científica y el desarrollo tecnológico guardan vinculación con el Estado. La investigación debe tener más un carácter vocacional, formando equipos de investigación, permitiendo el desarrollo de la misma en espacios y disciplinas menos favorecidos y con la ausencia de docentes-investigadores. Actualmente, las universidades quedan comprometidas con la enseñanza, la investigación, la creación y la difusión de los saberes. La investigación queda ligada e integrada al rol del docente (Algañaraz-Soria y Castillo, 2018).

Carvajal-Tapia y Carvajal-Rodríguez (2019) abordan la importancia del rol del docente en la enseñanza y en la investigación. Vale decir que la enseñanza, la investigación y el rol del docente permiten "la formación de recursos humanos idóneos, creativos, innovadores, apasionados". La enseñanza y la investigación son los pilares de la futura formación de los profesionales. En las universidades se percibe la necesidad "del desarrollo de estrategias para promover la investigación y formación de investigadores". El docente potencia la investigación en los estudiantes, en la formación de los futuros profesionales (Vásquez et al., 2020).

\section{$2.2 \quad$ Perfil cultural del docente}

\subsubsection{Aproximaciones conceptuales y referenciales}

Para Chenet-Zuta, Arévalo-Quijano y Palma-Alvino (2017), "la cultura es el conjunto de todas las formas y expresiones de una sociedad determinada. Como tal incluye costumbres, prácticas, códigos, normas y reglas de la manera de ser, vestimenta, religión, rituales, normas de comportamiento y sistemas de creencias" (pp. 302 y 303). 
El docente y su formación profesional depende de la triada: currículo, la educación y la cultura, incluso de la ciencia y la cultura. La cultura es imperativa en la formación del docente. En este sentido la relación formación-cultura es indisoluble, inquebrantable, la ligazón le da potencia a la formación cultural del docente (Rodríguez, 2017). Según la UNESCO (2015, citada por Ballesteros, Gracia, Ocaña y Jácome, 2018), la cultura es un conjunto cuyo contenido comprende "os rasgos distintivos, espirituales, materiales y afectivos" de un grupo social, de una comunidad, sociedad, nación. También se suman las artes, las letras, inclusive modos de vida, derechos fundamentales, valores, creencias, tradiciones.

Por su parte, Cely Galindo (2019) percibe que el perfil y la calidad del docente no queda al margen del enfoque antropológico y social, entendiendo que las conductas morales siempre constituyen constructos sociales muy importantes. Estas conductas generan cambios sociales y culturales, mejorando la calidad de la vida en la sociedad globalizada y las configuraciones sociales.

\subsubsection{Dimensiones del perfil cultural del docente}

La diversidad cultural

Las aulas y la sociedad muestran una realidad patente: la diversidad cultural, en la cual viven los padres y los hijos, quienes son sujetos y protagonistas en los escenarios de la educación. Existen grupos diversos por sus culturas, con la necesidad de integración multicultural (Rodríguez-Fuentes y Fernández-Fernández, 2018). La interculturalidad es una temática que es parte de las agendas político-pedagógicas, fomentando la educación intercultural bilingüe, descifrando e interpretando la investigación etnográfica, con el propósito vivir y construir la interculturalidad (Rodríguez-Cruz, 2018).

Para Castillo, Llorent, Salazar y Álamo (2018), los docentes interculturales bilingües son necesarios, quienes tienen las ventajas de alfabetizar en el lenguaje y en la cultura a los estudiantes, particularmente en los pueblos indígenas, los cuales poseen sus propias “características culturales y lingüísticas particulares". Los problemas lingüísticos y culturales son resueltos por los profesores interculturales bilingües. Es decir, ellos tienen la capacidad de realizar una educación bilingüe; tienen la capacidad de leer, analizar e interpretar los diversos códigos lingüísticos y culturales de los estudiantes y de la comunidad involucrada; ejercen una didáctica multicultural.

La interculturalidad significa la presencia mínima de dos culturas y dos lenguas; significa la relación sinérgica de las culturas anexas. Existen espacios, naciones y países multilingües y pluriculturales. En estos ambientes qué hacen los docentes, qué necesitan 
ellos para la educación de los estudiantes y de la comunidad. Las universidades tienen la responsabilidad de formar a docentes con perfiles interculturales bilingües. Es decir, el Estado y sus respectivas organizaciones tienen la obligación de organizar y sistematizar una educación intercultural bilingüe. Se requiere una comunicación, un diálogo intercultural en los procesos educativos y de enseñanza-aprendizaje. La interculturalidad es la "mediadora entre el estudiante, los docentes, la familia y la comunidad", en el espacio de la educación; es muy importante para educación multicultural, genera la relación entre culturales existentes dentro de la sociedad, las potencia y les genera la continuidad y la perpetuación (Aguavil-Arévalo y Andino-Jaramillo, 2019).

Difusión de la cultura

La cultura existe, pero quién la sabe, quién la difunde, dónde se difunde. García-Pérez, Herrera-Rodríguez, García-Valero y Guevara-Fernández (2015) responden estas interrogantes al declarar que las universidades "son las encargadas de preservar, desarrollar y difundir la cultura". En otras palabras, los protagonistas de la universidad, quienes son los docentes. Estos tienen la misión de difusión. Para ellos, "la cultura profesional docente colaborativa comprende al conjunto de conocimientos, actitudes, habilidades profesionales, hábitos, y valores que determinan la conducta del profesorado en el ejercicio de la función docente-metodológica, investigativa y orientadora, como resultado de un proceso de construcción colectiva" (p.6).

También es competencia cultural la visibilidad y difusión de las bibliotecas virtuales, propiciando la lectura y fortaleciendo la cultura y su conocimiento y uso (LázaroRodríguez, López-Gijón y Herrera-Viedma, 2018). La cultura no solamente se la observa, sino se la comparte, se la promociona. La promoción de la cultura se constituye en una herramienta poderosa, cuya existencia tiene un propósito: el fortalecimiento de la identidad, dejando la satisfacción con la misma en la experiencia de las personas involucradas con el conocimiento y la difusión. Ballesteros et al. (2018) afirman:

la promoción cultural está enfocada al crecimiento humano para intervenir en la relación entre entes reguladores que se encargan del desarrollo cultural y la sociedad. Desde el ámbito cultural la promoción se presenta como el resultado de tratar la cultura como un producto o valor que debe estar al alcance de su público objetivo (p. 370).

Identidad cultural

Chenet-Zuta, Arévalo-Quijano y Palma-Alvino (2017) aprecian que la identidad cultural mejora el desempeño docente en las instituciones educativas, constituye un recurso de formación docente, dándoles presencia y vigencia a las relaciones interculturales; mejora 
la vida y la cosmovisión cultural; genera la presencia de la multiculturalidad, promueve el desempeño intercultural, también el desarrollo en los aspectos intelectual y cultural.

La identidad cultural está ligada a la memoria, historia del pasado, los símbolos, estos constituyen los fundamentos históricos de los pueblos y las naciones, los cuales se conservan en la memoria para fortalecer la identidad (Ballesteros et al., 2018). Por otro lado, la identidad cultural no queda desligada de patrimonio, educación patrimonial, turismo cultural. Tiene relación directa con la educación y la gestión cultural, con las características autóctonas, la lengua, costumbres, folclor, bienes patrimoniales (Cepeda Ortega, 2018). Los mitos, las leyendas, el turismo cultural, la historia, no solamente constituyen patrimonio cultural de los pueblos y las naciones, sino la identidad cultural (Ortega-Alarcón, 2018).

Identidad nacional

La identidad nacional se construye mediante los medios de comunicación y las exposiciones selectivas. Son determinadas mediante las ideologías. También identidad referida apoya la defensa de la independencia y los valores sociodemográficos, los sentimientos de pertenencia cultural (Valera-Ordaz, 2018). Según Sonlleva-Velasco, Sanz-Simón y Torrego-Egido (2018), la identidad nacional se construye mediante la intervención de la iglesia, el Estado, también mediante la metodología denominada biográfico-narrativa; es decir, mediante narrativas de vida, elaboradas después de una guerra.

El sentimiento de pertenencia, la nación, la historia, la ciencia social, el Estado, las políticas exteriores, las imágenes nacionales, los actores, el interés social y nacional, los atributos nacionales, permiten la construcción de la identidad nacional (Tah Ayala, 2018). A veces la identidad nacional, en la experiencia de los pueblos, queda trabada. Entonces, aparecen las retóricas, las poéticas, los relatos históricos, los lenguajes poéticos, las narraciones, los lenguajes políticos y nacionales, con el propósito de desplazar, eliminar y destruir esas trabas que impiden la construcción de la identidad nacional (Uribe de Hincapié, 2019).

\section{Cultura innovadora}

Las universidades y los docentes tienen el compromiso de gestionar una cultura de innovación, medida y evaluada por expertos. La cultura tiene relación con la promoción de las actitudes, las habilidades, los valores, los conocimientos y las prácticas. Gestionar la innovación cultura significa habilitar a los sujetos en la condición de creativos, 
innovadores, facilitadores, activos, científicos, investigadores, extensionistas (NegrinReyes, 2018).

Según Donawa-Torres y Gámez-Araujo (2019), las instituciones y las empresas necesitan construir una cultura innovadora, con el propósito de desarrollar las habilidades gerenciales. En este sentido, todos los docentes asumen el compromiso de desarrollar sus habilidades gerenciales también las de sus discípulos. La cultura de la innovación se comparte, estimula el trabajo de la innovación. Todo el tiempo se necesitan, se buscan la innovación.

\section{Cultura organizacional}

Para Rivera-Porras et al., 2018), la cultura organizacional se ha constituido en un desafío, en un reto para las instituciones y organizaciones que salud, que son saludable. Tiene suma importancia para las organizaciones, cuyos participantes poseen conocimientos, capacidades, habilidades que generan la singularidad, en un proceso de cambio continuo. El docente responde a una malla curricular muy vasta. Es decir, el docente es formado y educado de acuerdo con "una malla compleja de dinámicas y decisiones sociopolíticas, culturales, pedagógicas y profesionales" (Escudero Muñoz, González González y Rodríguez Entrena, 2018). ¿Qué significa la cultura organizacional para la universidad, para el docente? La cultura organizacional tiene significativas repercusiones son el uso de las TICs. Estas se han constituido en factores de la cultura organizacional (Negrón, 2019).

\section{Conclusiones}

Luego de indagar sobre el perfil docente, en sus dimensiones educativa y cultural, se concluye que el perfil educativo del docente ofrece y proyecta una educación holística, inclusiva, acreditada, gamificada, emotiva e investigativa. El docente tiene la capacidad de atender, mediante el proceso enseñanza-aprendizaje, el desarrollo pleno de los estudiantes, el desarrollo del hombre en todas sus dimensiones, con la capacidad de inclusión, sin marginaciones de ningún tipo. También el docente revela su capacidad de uso de metodología motivadoras, generando emoción y calidad investigativa.

Por otro lado, el perfil del docente se constituye en na muestra de la diversidad cultural, de la difusión de la cultura, de la identidad cultural y nacional, de la cultura innovadora y organizacional. En efecto, el perfil cultural del docente significa la plenitud de la cultura, la restauración de la misma y su respectiva difusión, quien queda encantado con su cultura la comparte, la difunde. 
Referencias

Acosta, B. y Acosta, M. (2016). Modelos de evaluación para la acreditación de carreras. RMIE Revista Mexicana de Investigación Educativa, 21(21), 1249-1274. Retrieved from http://www.scielo.org.mx/pdf/rmie/v21n71/1405-6666-rmie-21-71-01249.pdf

Aguavil-Arévalo, J. M. y Andino-Jaramillo, R. A. (2019). Necesidades formativas de docentes de Educación Intercultural Tsáchila. Alteridad, 14(1), 74-83. Retrieved from https://doi.org/10.17163.alt.v14n1.2019.06

Algañaraz-Soria, V. H. y Castillo, G. M. (2018). Docencia e investigación en la Universidad Nacional de San Juan (1994-2015): impacto del programa de incentivos a docentes-investigadores y la carrera del investigador científico del CONICET. PROMETEICA - Revista de Filosofía y Ciencias, (16), 57-77. Retrieved from https://doi.org/10.24316/prometeica.v0i16.210

Alsina, Á., Batllori, R., Falgàs, M., \& Vidal, I. (2019). Marcas de autorregulación para la construcción del perfil docente durante la formación inicial de maestros. Revista Complutense de Educación, 30(1), 55-74. Retrieved from http://dx.doi.org/10.5209/RCED.55466

Arroyo-González, M. J. (2013). La Educación Intercultural: un camino hacia la inclusión educativa. Revista de Educación Inclusiva, 6(2), 144-159. Retrieved from https://dialnet.unirioja.es/servlet/articulo?codigo $=4335836$

Ballesteros, C., Gracia, G., Ocaña, A. y Jácome, C. (2018). Análisis de la promoción cultural como herramienta para fortalecer la identidad afro esmeraldeña. Revista Lasallista de Investigación, 15(2), 367-377. https://doi.org/10.22507/rli.v15n2a28

Barrientos-Gutiérrez, P. (2016). La naturaleza de la formación docente. Horizonte de la Ciencia, $6(11)$ $169-177$. https://doi.org/https://doi.org/10.26490/uncp.horizonteciencia.2016.11.238

Bisquerra, R. y Hernández, S. (2017). Psicología positiva, educación emocional y el programa aulas felices. Papeles del Psicólogo, 38(1), 58-65. Retrieved from https://doi.org/10.23923/pap.psicol2017.2822

Carvajal-Tapia, A. E. y Carvajal-Rodríguez, E. (2019). La importancia del rol docente en la enseñanza e investigación The importance of the teaching role in teaching and research. RIP, 107-114. Retrieved from http://www.scielo.org.bo/pdf/rip/n21/n21_a08.pdf 
Castillo, I., Llorent, V. J., Salazar, L. y Álamo, M. (2018). Lenguaje , diversidad cultural y currículo. Perfiles Educativos, XL(162), 54-67. Retrieved from http://www.scielo.org.mx/pdf/peredu/v40n162/0185-2698-peredu-40-162-54.pdf

Cely Galindo, G. (2019). Acerca de la moral y la sabiduría. Cuadernos de Contabilidad, 20(49), 1-8. https://doi.org/https://doi.org/10.11144/Javeriana.cc20-49.adms

Cepeda Ortega, J. (2018). Una aproximación al concepto de identidad cultural a partir de experiencias: el patrimonio y la educación. Tabanque, 31, 244-262. https://doi.org/https://doi.org/10.24197/trp.31.2018.244-262

Chenet-Zuta, M. E., Arévalo-Quijano, J. C. y Palma-Alvino, F. (2017). Identidad cultural y desempeño docente en instituciones educativas. Revista de Ciencias Humanas Y Sociales, 33(84), 292-322. Retrieved from https://produccioncientificaluz.org/index.php/opcion/article/view/23390/23573

Clavijo, R. G. y Bautista, M. J. (2020). La educación inclusiva. Análisis y reflexiones en la educación superior ecuatoriana. Alteridad, 15(1), 113-124. https://doi.org/https://doi.org/10.17163/alt.v15n1.2020.09

Corchuelo-Rodriguez, C. A. (2018). Gamificación en educación superior: experiencia innovadora para motivar estudiantes y dinamizar contenidos en el aula. EDUTEC. Revista Electrónica de Tecnología Educativa, (63), 29-41. https://doi.org/dx.doi.org/10.21556/edutec.2018.63.927

Corredor, Ó.y Saker, J. (2018). Perspectiva de la formación científica de docentes en instituciones de educación básica y media - Barranquilla Perspective of the scientific training of teachers in basic and media education institutions - Barranquilla. Educación y Humanismo, 20(34), 156-172. Retrieved from https://doi.org/10.17081/eduhum.20.34.2862

Díez-Gutiérrez, E. J. (2018). Universidad e investigación para el bien común: la función social de la universidad. Aula Abierta, 47(4), 395-402. https://doi.org/https://doi.org/10.17811/rifie.47.4.2018.395-402

Dimas-Rangel, M. I.y Treviño-Sánchez, A. (2015). La importancia de la definición del perfil docente de una institución de educación superior en ingeniería. Vincula Tégica, 1(1), 1662-1680. Retrieved from http://eprints.uanl.mx/17298/1/93.pdf

Donawa-Torres, Z. A.y Gámez-Araujo, W. G. (2019). Ausencia de habilidades gerenciales para una cultura de innovación universitaria en instituciones de Colombia y Venezuela. Universidad y Empresa, 21(36), 8-35. https://doi.org/http://dx.doi.org/10.12804/revistas.urosario.edu.co/empresa/a.5961 
Escudero Muñoz, J. M., González González, M. T.y Rodríguez Entrena, M. J. (2018).

Los contenidos de la formación continuada del profesorado: ¿Qué docentes se están formando? Educación XX1, 2(1), 157-180. https://doi.org/10.5944/educXX1.15807

Florian, L. (2014). La educación especial en la era de la inclusión: ¿El fin de la educación especial o un nuevo comienzo? Special education in the era of inclusion: The end of special education or a new beginning? The Psychology of Education Review, 34(2), 2736.

Retrieved from http://www.repositoriocdpd.net:8080/bitstream/handle/123456789/1705/Art_FlorianL_ Educacionespecial_2013.pdf?sequence=1

Galvis, R. V. (2007). De un perfil docente tradicional a un perfil docente basado en competencias. Acción Pedagógica, (16), 48-57. Retrieved from http://www.comie.org.mx/congreso/memoriaelectronica/v14/doc/1135.pdf

García-Pérez, Y., Herrera-Rodríguez, J. I., García-Valero, M. de los Á. y GuevaraFernández, G. E. (2015). El trabajo colaborativo y su influencia en el desarrollo de la cultura profesional docente. Gaceta Médica Espirituana, 17(1), 1-7. Retrieved from http://scielo.sld.cu/pdf/gme/v17n1/GME06115.pdf

Hernández-Mosqueda, J. S., Tobón-Tobón, S. y Vázquez-Antonio, J. M. (2014). Estudio conceptual de la docencia socioformativa. Ra Ximhai, 10(5), 89-101. Retrieved from http://www.redalyc.org/articulo.oa?id=46132134006

Jiménez-Hernández, D., Sancho-Requena, P. y Sánchez-Fuentes, S. (2019). Perfil del futuro docente: nuevos retos en el marco del EEES. Contextos Educativos, (23), 125139. https://doi.org/http://doi.org/10.18172/con.3471 PERFIL

Jiménez, J. A. (2019). La evaluación y acreditación de la educación profesional en México: ¿la legitimación y competitividad como fin de la universidad? RESU Revista de La Educación Superior, 48(189), 55-72. Retrieved from http://www.scielo.org.mx/pdf/resu/v48n189/0185-2760-resu-48-189-55.pdf Lázaro-Rodríguez, P., López-Gijón, J. y Herrera-Viedma, E. (2018). Visibilidad de las bibliotecas públicas y la lectura en medios de comunicación españoles frente a otros hechos de la cultura y relación con su uso: medidas para su mayor promoción y difusión. BID: Textos Universitaris de Biblioteconomia i Documentación, 40(junio), 1-14. https://doi.org/https://dx.doi.org/10.1344/BiD2018.40.7

Leiva-Olivencia, J. J. y Gómez-Gerdel, M. de los Á. (2015). La educación inclusiva como constructo pedagógico en el alumnado universitario de educación primaria. Revista Nacional E Internacional de Educación Inclusiva, 8(2), 185-200. 
Lozano-González, E. O. (2017). La cultura y la formación docente en la vida cotidiana escolar, $1-10$.

Retrieved

from

http://www.comie.org.mx/congreso/memoriaelectronica/v14/doc/1135.pdf

Márquez, E. y Zeballos-Pinto, Z. R. (2017). El impacto de la acreditación en la mejora de la calidad de los programas educativos que ofrece la Universidad Autónoma de Tamaulipas: un estudio de caso. Revista Iberoamericana de Evaluación Educativa, 10(2), 65-83. https://doi.org/https://doi.org/10.15366/riee2017.10.2.004

Martínez-Iñiguez, J. E., Tobón, S. y Romero-Sandoval, A. (2017). Problemáticas relacionadas con la acreditación de la calidad de la educación superior en América Latina. Innovación Educativa, 17(73), 79-96.

Maturana, G. y Mahecha, L. M. (2020). Las modalidades de educación a distancia y virtual en la formación posgradual: Una estrategia para la educación inclusiva en América Latina. Educación Superior y Sociedad, 32, 36-58. Retrieved from https://www.iesalc.unesco.org/ess/index.php/ess3/article/view/232/212

Melgar, M. F. y De Los Reyes Leoz, J. L. (2018). De los objetos al museo. Experiencias educativas emotivas y creativas. Contextos de Educación, 20(25), 122-137. Retrieved from www.hum.unrc.edu.ar/ojs/index.php/contextos

Melo-Solarte, D. S.,y Díaz, P. A. (2018). El Aprendizaje Afectivo y la Gamificación en Escenarios de Educación Virtual Emotional Learning and Gamification in Virtual Education Environments. Información Tecnológica, 29(3), 237-248. https://doi.org/http://dx.doi.org/10.4067/S0718-07642018000300237

Montes, I. C. y Mendoza, P. (2018). Docencia e investigación en Colombia desde la perspectiva del capitalismo académico. Archivos Analíticos de Políticas Educativas, 26(40), 1-30. Retrieved from http://dx.doi.org/10.14507/epaa.26.3220

Moreto, G., González-Blasco, P. y Piñero, A. (2018). Reflexiones sobre la deshumanización de la educación médica: empatía, emociones y posibles recursos pedagógicos para la educación afectiva del estudiante de medicina. Educación Médica, 19(3), 172-177. https://doi.org/10.1016/j.edumed.2016.12.013

Muntaner-Guasp, J. J., Rosselló-Ramón, M. R. y De La Iglesia-Mayol, B. (2016). Buenas prácticas en educación inclusiva Good practices in inclusive education. Educatio Siglo XXI, 34(1), 31-50. Retrieved from http://dx.doi.org/10.6018/j/252521

Negrin-Reyes, D. E. (2018). Procedimiento para gestionar la cultura de innovación de los cuadros docentes de la Universidad de Pinar del Río. Universidad de Pinar del Río "Hermanos Saiz Montes de Oca". 
Negrón, S. (2019). Influencia de la cultura organizacional universitaria en el uso de las

TIC. Revista Electrónica de Investigación y Docencia (REID), (21), 67-80. https://doi.org/10.17561/reid.n21.5

Ortega-Alarcón, A. E. (2018). Difusión de los “mitos y leyendas” como herramienta para desarrollar el turismo cultural del cantón Babahoyo, provincia de Los Ríos. [Tesis, Universidad Técnica de Babahoyo]. Retrieved from http://dspace.utb.edu.ec/bitstream/handle/49000/4241/P-UTB-FCJSE-HTURIS000037.pdf? sequence $=1$

Ortiz-Colón, A.-M., Jordán, J. y Agredal, M. (2018). Gamificación en educación: una panorámica sobre el estado de la cuestión. Educ. Pesqui., São Paulo, 44, 1-17. https://doi.org/http://dx.doi.org/10.1590/S1678-4634201844173773

Parra-González, M. E. y Segura-Robles, A. (2019). Producción científica sobre gamificación en educación : un análisis cienciométrico Scientific production about gamification in education : A Scientometric analysis. Revista de Educación, (386), 113135. https://doi.org/10.4438/1988-592X-RE-2019-386-429

Prieto-Egido, M. (2018). La psicologización de la educación: Implicaciones pedagógicas de la inteligencia emocional y la psicología positiva. [The psychologisation of education: Educational implications of emotional intelligence and positive psychology]. Educación XX1, 21(1), 303-320. https://doi.org/10.5944/educXX1.16058

Rivera, D. A., Carrillo, S. M., Forgiony, J. O., Nuván, I. L. y Rozo, A. C. (2018). Cultura organizacional, retos y desafíos para las organizaciones saludables. Espacios, 39(22), 114.

Retrieved

from

http://bonga.unisimon.edu.co/bitstream/handle/20.500.12442/2165/Cultura

organizacional.pdf? sequence $=1 \&$ isAllowed $=\mathrm{y}$

Rodríguez-Cruz, M. (2018). Construir la interculturalidad. Políticas educativas, diversidad cultural y desigualdad en Ecuador. Íconos. Revista de Ciencias Sociales, (60), 217-236. https://doi.org/http://dx.doi.org/10.17141/iconos.60.2018.2922

Rodríguez-Fuentes, A. y Fernández-Fernández, A. D. (2018). Actitudes ante la diversidad cultural de progenitores y descendientes. Eficiencia de la influencia por su grado de confluencia. Arbor, 194(788), 1-15. Retrieved from https://doi.org/10.3989/arbor.2018.788n2011

Rodríguez, M. (2017). Currículum, educación y cultura en la formación docente del siglo XXI desde la complejidad. Revista Educación Y Humanismo, 19(33), 425-440. https://doi.org/http://dx.doi.org/10.17081/eduhum.19.33.2654 
Smith, P.y Smith, J. (2018). Investigar en Educación. Conceptos básicos y metodología para desarrollar proyectos de investigación. Educatio Siglo XXI, 36(1), 263-266.

Sonlleva-Velasco, M., Sanz-Simón, C. y Torrego-Egido, L. (2018). El retrato de Franco, el de José Antonio y el crucifijo. Construcción de la identidad nacional en los escolares de posguerra. El Futuro del Pasado, (9), 331-363. https://doi.org/http://dx.doi.org/10.14516/fdp.2018.009.001.012

Tah Ayala, E. D. (2018). Las relaciones internacionales desde la perspectiva social. La visión del constructivismo para explicar la identidad nacional. Revista Mexicana de

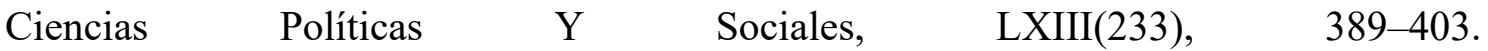
https://doi.org/10.22201/fcpys.2448492xe.2018.233.62593

Uribe de Hincapié, M. T. (2019). La elusiva y difícil construcción de la identidad nacional en la Gran Colombia. Co-Herencia, 16(31), 13-44. https://doi.org/10.17230/coherencia.16.31.1

Valera-Ordaz, L. (2018). Medios, identidad nacional y exposición selectiva: predictores de preferencias mediáticas de los catalanes. Revista Española de Investigaciones Sociológicas, (164), 135-154. https://doi.org/10.5477/cis/reis.164.135

Vásquez, S., Vásquez, S. A., Vásquez, C. A., Alania, R., Díaz, M. y del Águila, M. (2020). El perfil del docente investigador: hacia sus dimensiones y fortalecimiento The profile of the research teacher: towards its dimensions and its strengthening. Apuntes Universitarios, 10(4), 69-88. https://doi.org/https://doi.org/10.17162/au.v10i4.492 Velasco-Cruz, S. (2016). Racismo y educación en México Racism and Education in Mexico. Revista Mexicana de Ciencias Políticas y Sociales, 61(226), 379-407. https://doi.org/10.1016/S0185-1918(16)30015-0 